\title{
Quantitative measurement of the outer membrane permeability in Escherichia coli Ipp and tol-pal mutants defines the significance of Tol-Pal function for maintaining drug resistance
}

\author{
Hikaru Kowata $^{1}$, Saeko Tochigi ${ }^{1}$, Tomonobu Kusano ${ }^{1}$ and Seiji Kojima ${ }^{1,2}$
}

Ensuring the stability of the outer membrane permeability barrier is crucial for maintaining drug resistance in Gram-negative bacteria. Lpp protein and Tol-Pal complex are responsible for this function and are widely distributed among Gram-negative bacteria. Thus, these proteins are potential targets to permeabilize the outer membrane barrier. Although deleting these proteins is known to impair the outer membrane stability, the effect of the deletion on the outer membrane barrier property and on the drug resistance has not been fully characterized and evaluated in a quantitative manner. Here, we determined the outer membrane permeability of Escherichia coli $\Delta / p p$ and $\Delta$ tol-pal mutants by the assay using intact cells and liposomes reconstituted with the outer membrane proteins. We determined that there was 3- to 5-fold increase of the permeability in $\Delta$ tol-pal mutants, but not in $\Delta / p p$ mutant, compared with that in the parental strain. The permeability increase in $\Delta$ tol-pal mutants occurred without affecting the function of outer membrane diffusion channels, and was most pronounced in the cells at exponential growth phase. The impact of tol-pal deletion on the drug resistance was revealed to be almost comparable with that of deletion of $a c r A B$, a major multidrug efflux transporter of $E$. coli that makes a predominant contribution to drug resistance. Our observations highlight the importance of Tol-Pal as a possible target to combat multidrug-resistant Gram-negative bacteria. The Journal of Antibiotics (2016) 69, 863-870; doi:10.1038/ja.2016.50; published online 11 May 2016

\section{INTRODUCTION}

The outer membrane of Gram-negative bacteria serves as a permeability barrier that prevents the entry of noxious compounds into the cell, ${ }^{1}$ thereby having the crucial role in multidrug resistance. Ensuring the barrier function of the outer membrane requires not only appropriate biogenesis of the outer membrane, that is, the biosynthesis, transport and assembly of outer membrane components such as lipids and proteins, ${ }^{2,3}$ but also structural proteins that maintain its stability and integrity. Murein-lipoprotein (Lpp) and the Tol-Pal complex are widely conserved in Gram-negative bacteria and form a physical connection between the outer membrane and peptidoglycan, thereby maintaining the structural stability of the outer membrane. ${ }^{4-9}$ Lpp is a small outer membrane lipoprotein that consists of 58 amino acids and is the most abundant outer membrane lipoprotein. About one-third of Lpp molecules are covalently linked to the peptidoglycan, and the remaining exist as free form. ${ }^{9}$ The Tol-Pal complex forms the structural bridge between the outer membrane and the cytoplasmic membrane. ${ }^{10-12}$ It comprises (at least) five proteins: TolA, TolQ and TolR form the cytoplasmic membrane component, TolB is a periplasmic protein and $\mathrm{Pal}$ is an outer membrane lipoprotein. Mutants defective in Lpp or any component of the Tol-Pal complex are known to show an increased susceptibility to drugs, leakiness of periplasmic proteins and partial detachment of the outer membrane from peptidoglycan, resulting in the formation of blebs or vesicles. ${ }^{7,8,13}$ Although the increased drug susceptibility of $\Delta l p p$ and $\Delta t o l-p a l$ mutants suggest that these proteins can be the potential target to permeabilize the outer membrane and therefore to combat multidrugresistant Gram-negative bacteria, the significance of Lpp and Tol-Pal function on the outer membrane permeability and the drug resistance has not been fully characterized in a quantitative manner. Here, we determined the outer membrane permeability of these mutants by the assay using intact cells and liposomes reconstituted with the outer membrane proteins. We determined that there was 3- to 5-fold increase of the permeability in $\Delta t o l-p a l$ mutants, but not in $\Delta l p p$ mutant, compared with that in parental strain. The permeability increase in $\Delta$ tol-pal mutants occurred without affecting the function of outer membrane diffusion channels, and was most pronounced in the cells at exponential growth phase. The impact of tol-pal deletion on the drug resistance was revealed to be almost comparable with that of deletion of $a c r A B$, a major multidrug efflux transporter of $E$. coli that makes a predominant contribution to drug resistance. Our observations highlight the importance of Tol-Pal as a possible target to combat multidrug-resistant Gram-negative bacteria.

${ }^{1}$ Graduate School of Life Sciences, Tohoku University, Sendai, Japan and ${ }^{2}$ Frontier Research Institute for Interdisciplinary Sciences, Tohoku University, Sendai, Japan Correspondence: Dr S Kojima, Graduate School of Life Sciences, Tohoku University, Katahira 2-1-1, Aoba-ku, Sendai 9808577, Japan.

E-mail: skojima0801@gmail.com

Received 20 January 2016; revised 16 March 2016; accepted 5 April 2016; published online 11 May 2016 


\section{MATERIALS AND METHODS}

\section{Bacterial strains}

LA51 is an E. coli K-12 derivative harboring a mutation in the promoter region of AmpC $\beta$-lactamase, which upregulates AmpC expression. ${ }^{14}$ The construction of the LA51 $\triangle a c r A B$ mutant was described previously. ${ }^{15}$ LA51 mutants, in which the tolA, tolB, tolQ, tolR, pal or lpp genes are deleted, were constructed by transducing disrupted gene cassette containing $\mathrm{Km}^{\mathrm{r}}$ from JW0729, JW5100, JW0727, JW0728, JW0731 or JW1667 (Keio collection), ${ }^{16}$ respectively. The transductants were selected with $35 \mu \mathrm{g} \mathrm{ml}^{-1}$ kanamycin. To construct the LA51 $\triangle a c r A B$ double mutants with tol-pal or lpp deletion, we first transduced $a c r A B:: \mathrm{Km}^{\mathrm{r}}$ gene from RAM1337, ${ }^{17}$ and the kanamycin-resistant gene was removed using pCP20 according to the method of Datsenko and Wanner. ${ }^{18}$ Next, the tol-pal or lpp genes were deleted as described above. The transduction was performed using the $\mathrm{P} 1 \mathrm{cml}, \mathrm{clr} 100$ phage and standard protocols. ${ }^{19}$

\section{Measurement of outer membrane permeability}

The protocol was essentially the same as described previously. ${ }^{15,20}$ Briefly, the cells were grown in $50 \mathrm{ml}$ of LB medium supplemented with $5 \mathrm{~mm} \mathrm{MgCl}_{2}$ at $37^{\circ} \mathrm{C}$ with shaking, and collected at an $\mathrm{OD}$ at $600 \mathrm{~nm}\left(\mathrm{OD}_{600}\right)$ of $1 \sim 1.2$ by centrifugation at $5000 \mathrm{~g}$ for $10 \mathrm{~min}$ at room temperature (RT). The cells were washed once with $50 \mathrm{~mm}$ potassium-phosphate buffer ( $\mathrm{pH}$ 7.0) containing $5 \mathrm{~mm} \mathrm{MgCl}_{2}$ (K-P buffer) and suspended in the same buffer. The $\mathrm{OD}_{600}$ of the suspension was adjusted to 4.0 (corresponding to $1.5 \mathrm{mg}$ (dry cell weight) per $\mathrm{ml}$ ). To measure the influx of benzylpenicilliin (PEN), the cell suspension was mixed with various concentrations of PEN and incubated at RT for $10 \mathrm{~min}$, after which the mixture was heated in a boiling water bath for 1 min to stop hydrolysis by AmpC. During this period, any PEN that permeated outer membrane was subsequently hydrolyzed by AmpC. The amount of hydrolyzed PEN was quantified by microiodometry. ${ }^{21}$ The net rate of influx $\left(V_{\text {in }}\right)$ was calculated as the rate of hydrolysis $\left(V_{\mathrm{h}}\right)$ per milligram dry cell weight. It should be noted that when $a c r A B^{+}$strains are used, the $V_{\text {in }}$ value is affected by the rate of efflux $\left(V_{\mathrm{e}}\right)$. Accordingly, $\triangle a c r A B$ strains (with additional deletion of tol-pal or lpp genes) were used to determine the permeability coefficient $(P)$ of the outer membrane. $P$ was calculated using Fick's first law of diffusion: $V_{\text {in }}=P \cdot A \cdot\left(C_{\mathrm{o}}-C_{\mathrm{p}}\right)$, where $A, C_{0}$, and $C_{\mathrm{p}}$ represents the cell surface area $\left(132 \mathrm{~cm}^{2} \mathrm{mg}^{-1}\right.$ (dry cell weight)) for E. coli, ${ }^{22}$ extracellular and periplasmic concentrations of PEN, respectively. $C_{\mathrm{p}}$ was calculated using Michaelis-Menten equation: $C_{\mathrm{p}}=V_{\mathrm{h}} \times K_{\mathrm{m}} /\left(V_{\max }-V_{\mathrm{h}}\right)$, where $V_{\max }$ and $K_{\mathrm{m}}$ are kinetic parameters for $\mathrm{AmpC}$, which were determined using cell extract as described previously. ${ }^{15}$

Nitrocefin permeability was examined by mixing $50 \mu \mathrm{M}$ nitrocefin with the cell suspension prepared as described above. Hydrolysis of nitrocefin results in the increase in absorbance at $486 \mathrm{~nm}\left(A_{486}\right)$, and the complete hydrolysis of $100 \mu \mathrm{M}$ nitrocefin gives the increase of $A_{486}$ by 2.05. The $A_{486}$ of the reaction mixture was monitored for $2 \mathrm{~min}$, and hydrolysis rate, which is equal to $V_{\mathrm{in}}$, was calculated.

\section{Measurement of the leakage of periplasmic $\beta$-lactamase}

The cell suspensions prepared as above were incubated at RT for $15 \mathrm{~min}$ and the supernatant was collected after pelleting the cells by centrifugation at $20000 \mathrm{~g}$ for $5 \mathrm{~min}$ at RT. The supernatant was then mixed with $50 \mu \mathrm{M}$ PEN and incubated at RT for $10 \mathrm{~min}$. The amount of hydrolyzed PEN was quantified by microiodometry.

\section{MIC determination}

The protocol was the same as previously described. ${ }^{15}$ Briefly, approximately $10^{4}$ cells were inoculated to liquid LB broth or linear gradient plates containing drugs to be tested, and incubated for $18 \mathrm{~h}$ at $37^{\circ} \mathrm{C}$.

\section{Preparation of outer membrane proteins}

The protocol was the same as described previously. ${ }^{20}$

\section{Liposome swelling assay}

The protocol was essentially the same as described by Nikaido et al. ${ }^{23}$ Briefly, $2 \mu \mathrm{g}$ of outer membrane proteins were reconstituted into liposomes comprising $2.4 \mu \mathrm{mol}$ of phosphatidylcholine and $0.15 \mu \mathrm{mol}$ of dicetylphosphate. In this assay, the influx of substrates into the proteoliposome causes the proteoliposome to swell, resulting in the reduction in the absorbance $\left(\mathrm{at}^{\mathrm{O}} \mathrm{D}_{400}\right)$ of the reaction mixture. Thus, the rate swelling, that is, the rate at which the $\mathrm{OD}_{400}$ decreases upon mixing of the proteoliposome with substrates, is taken as the rate of influx.

\section{Electron microscopy}

The samples were prepared according to the protocol of Fung et al. ${ }^{24}$ with several modifications. The cells were collected from $50 \mathrm{ml}$ of LB medium (at $\mathrm{OD}_{600} \sim 1$ ) by centrifugation at $400 \mathrm{~g}$ for $10 \mathrm{~min}$ at RT, washed once with K-P buffer, and suspended in the same buffer. The cell suspension was then mixed with $2 \%$ (final concentration) of glutaraldehyde, and incubated at RT for $1.5 \mathrm{~h}$. The cells were pelleted by centrifugation at $400 \mathrm{~g}$ for $10 \mathrm{~min}$ at RT, and then embedded in $2 \%$ agar. After the agar solidified, it was washed with K-P buffer and soaked in $1 \%$ osmium tetroxide in Veronal buffer for $1 \mathrm{~h}$ at RT. The agar blocks were then dehydrated by soaking in increasing concentrations of ethanol $(60,70,80,90$ and $100 \%$ for 30 min each). The agar blocks were then soaked in propylene oxide for $30 \mathrm{~min}$ at RT. This step was repeated three times to completely replace the ethanol with propylene oxide. The blocks were then embedded in Quetol651 (Nisshin EM, Tokyo, Japan) and the samples were thin-sectioned $(60 \mathrm{~nm})$ using an ultramicrotome and a diamond knife. The samples were stained with Ti blue (Nisshin EM; diluted 10-fold) and 0.4\% lead citrate. The samples were observed under a transmission electron microscope (H-7650 Hitachi, Tokyo, Japan) at an accelerating voltage of $100 \mathrm{kV}$.

\section{Theoretical calculation of the movement and concentration of PEN across the outer membrane}

When $\beta$-lactam antibiotics are added to E. coli cells, they penetrate the outer membrane and then undergo either efflux transport via AcrAB-TolC or hydrolysis via AmpC $\beta$-lactamase. In a steady state, the relationship between $V_{\text {in }}, V_{\mathrm{e}}$ and $V_{\mathrm{h}}$ is described by the following equation: $V_{\mathrm{in}}=V_{\mathrm{e}}+V_{\mathrm{h}}$ (equation (1)). Previous study showed that this steady-state assumption was applicable to explain the movement of $\beta$-lactam antibiotics across the outer membrane. ${ }^{15}$ For PEN, all of the kinetic parameters required to describe $V_{\mathrm{in}}, V_{\mathrm{h}}$ and $V_{\mathrm{e}}$ are available, and these can be described as follows: $V_{\text {in }}=P \cdot A \cdot\left(C_{\mathrm{o}}-C_{\mathrm{p}}\right)$, Fick's first law of diffusion; $V_{\mathrm{h}}=V^{\mathrm{h}}{ }_{\max } \times K_{\mathrm{m}} /\left(K_{\mathrm{m}}+C_{\mathrm{p}}\right)$, the Michaelis-Menten equation; and $V_{\mathrm{e}}=V_{\text {max }}^{\mathrm{e}}{ }^{\cdot} C_{\mathrm{p}}{ }^{h} /\left(K_{0.5}{ }^{h}+C_{\mathrm{p}}{ }^{h}\right)$, the Hill equation, in which $V_{\max }^{\mathrm{e}}, K_{0.5}$ and $h$ are the maximum rate of efflux that AcrAB-TolC can produce, the substrate concentration that yields half-maximal efflux rate and the Hill coefficient, respectively. The parameters for PEN used in this theoretical analysis were as follows: $P=0.07 \times 10^{-5} \mathrm{~cm} \mathrm{~s}^{-1}, V_{\max }^{\mathrm{e}}=0.085 \mathrm{nmol} \mathrm{mg}^{-1} \mathrm{~s}^{-1}, K_{0.5}=0.30 \mu \mathrm{M}$, $h=4.0, V^{h}{ }_{\max }=0.330 \mathrm{nmol} \mathrm{mg}^{-1} \mathrm{~s}^{-1}$ and $K_{\mathrm{m}}=5.28 \mu \mathrm{M} .{ }^{15}$ Substituting these parameters into equation (1) gives the function of $C_{\mathrm{p}}$ against $C_{\mathrm{o}}$, which was used to create the theoretical $C_{\mathrm{p}}$ versus $C_{\mathrm{o}}$ curve. Using this function, the influence of an increase in outer membrane permeability was assessed by substituting 4-fold higher value of $P\left(0.28 \times 10^{-5} \mathrm{~cm} \mathrm{~s}^{-1}\right)$.

\section{RESULTS}

Measurement of the outer membrane permeability in $\Delta$ tol-pal and $\Delta l p p$ mutants using the intact cells

Although both of $\Delta l p p$ and $\Delta$ tol-pal mutants are known to impair the outer membrane stability, scrutiny of their phenotype highlights the several lines of evidence that suggest the difference in their outer membrane barrier property. (1) Lpp deletion increases susceptibility to drugs mainly those with high hydrophobicity, whereas there is no apparent change in the MIC for relatively hydrophilic drugs, such as ampicillin. ${ }^{7}$ Also, the permeation rate of 6-aminopenicillanic acid across the outer membrane was shown to be unaltered by the deletion of Lpp. ${ }^{25}$ By contrast, deleting either of the tol-pal genes increases the susceptibility to hydrophobic as well as hydrophilic drugs that even include vancomycin, which is usually excluded by the outer membrane as it is both large and hydrophilic. ${ }^{26}$ These susceptibility patterns are also supported by a study by Nichols et al. ${ }^{27}$ which quantified the growth phenotype of nearly 4000 E. coli deletion mutants (Keio 
collection) in the presence of various drugs. (2) The addition of $\mathrm{Mg}^{2+}$, a divalent cation known to stabilize the outer membrane by reinforcing the lateral interactions between lipopolysaccharide (LPS) molecules, ${ }^{1}$ to the growth medium represses bleb or vesicle formation in $l_{p p}$ mutant, ${ }^{24}$ whereas tol-pal mutants are not apparently responsive to the external $\mathrm{Mg}^{2+}$ concentration. ${ }^{28,29}$
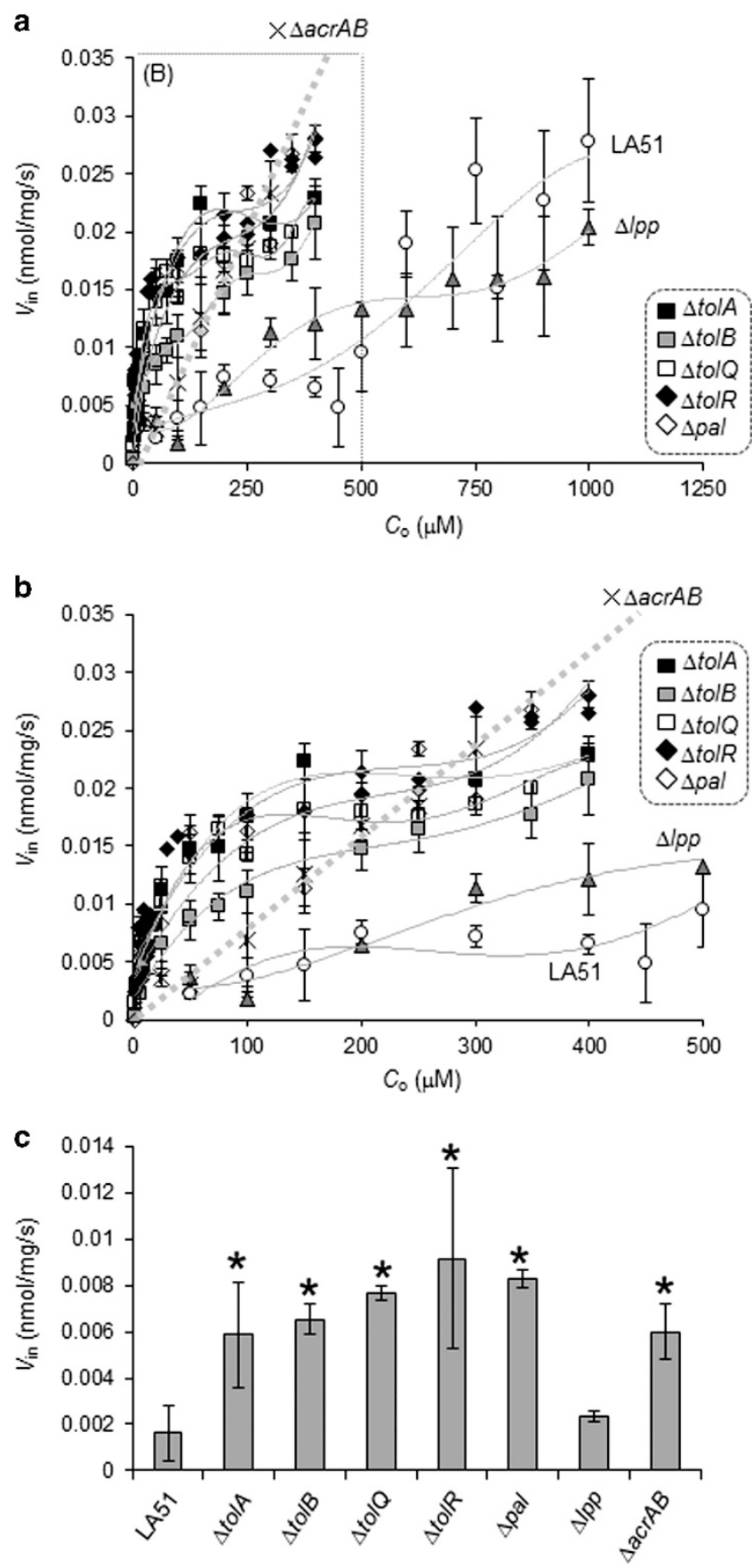

Figure 1 Rate of PEN influx into LA51 cells and derivative mutant cells. (a) $C_{0}$ is the extracellular PEN concentration. For clarity, a fitted line is shown to reflect the approximate $V_{\text {in }}$ for the $\triangle a c r A B$ strain. Data obtained below $500 \mu \mathrm{m}$ of $C_{0}$ is shown in an enlarged graph (b). (c) Rate of nitrocefin influx into LA51 cells and its derivative mutants cells. Concentration of nitrocefin was $50 \mu \mathrm{m}$. Asterisks show that the difference compared with LA51 was statistically significant according to the Student's $t$-test $(P<0.05)$. The data are expressed as the mean \pm s.d. of triplicate measurements.
To evaluate the outer membrane permeability of the $\Delta$ tol-pal and $\Delta l_{p p}$ mutant, we measured the influx rates of PEN across the outer membrane of intact cells by coupling it with subsequent hydrolysis by periplasmic AmpC $\beta$-lactamase, a reaction that can be experimentally quantified according to the method of Kojima and Nikaido. ${ }^{15}$ For this assay, the net rate of PEN influx $\left(V_{\text {in }}\right)$ was determined as its rate of hydrolysis $\left(V_{\mathrm{h}}\right)$. PEN was chosen as a substrate because (i) it is sensitive enough to AmpC hydrolysis, ${ }^{30}$ so $V_{\mathrm{h}}$ can be easily quantified and is expected to reflect precise differences in outer membrane permeability, even if they are only marginal and (ii) PEN diffuses through porin channels, but because it is not too hydrophilic, it slowly penetrates the phospholipid bilayer; ${ }^{31}$ therefore, possible outer membrane $\operatorname{defect}(\mathrm{s})$ in the lipid bilayer region and in proteinaceous channels are expected to be reflected by $V_{\text {in. }}$. A prerequisite for the assay was that the cells did not leak a significant amount of $\beta$-lactamase into the external environment during the experiment. We examined $\beta$-lactamase activity in the supernatant after suspending cells in buffer at RT. We confirmed that using a buffer supplemented with $5 \mathrm{mM} \mathrm{MgCl}_{2}$ prevented significant $\beta$-lactamase leakage, at least within the 15-min incubation period (Supplementary Table S1). The rate of PEN influx into LA51 (parental strain), $\Delta$ tol-pal and $\Delta l p p$ cells is shown in Figure 1. Deleting any component of the Tol-Pal complex led to marked increase of $V_{\text {in }}$. By contrast, we observed no detectable difference of $V_{\text {in }}$ in $\Delta l p p$ mutant. The increase in $V_{\text {in }}$ in $\Delta$ tol-pal mutants was substantial, and this led us to compare it with the $V_{\text {in }}$ for a mutant lacking $\mathrm{Acr} A \mathrm{~B}$, an efflux transporter that pumps out a broad range of drugs from the periplasm (or inner membrane) and makes a predominant contribution to the multidrug efflux activity of E. coli. ${ }^{32}$ We found that the $V_{\text {in }}$ for $\Delta$ tol-pal mutants was comparable with that for $\triangle a c r A B$ mutant (Figures 1a and b). The sigmoidal nature of the data, which was apparent in all the strains except $\triangle a c r A B$ mutant, was derived from the positive cooperativity of the efflux activity by AcrABTolC. ${ }^{15,33}$ To obtain the value for the permeability coefficient $(P)$, a robust index that can be used to quantify outer membrane permeability, we constructed double deletion mutants ( $\triangle$ tol-pal/ $\triangle a c r A B$ or $\Delta l p p / \triangle a c r A B)$ to eliminate the influence of efflux activity on $V_{\mathrm{in}}$. We observed a 3- to 5 -fold increase in $P$ for the $\Delta$ tol-pal mutants, but not for the $\Delta l p p$ mutants (Table 1). To clarify whether similar increase of permeability occurs with other class of $\beta$-lactam antibiotics, we tested the permeability of nitrocefin (Figure 1c). The $V_{\text {in }}$ of nitrocefin in $\Delta$ tol-pal and $\triangle a c r A B$ was markedly increased compared with that in parental strain, whereas only modest increase was observed in $\Delta l p p$ mutant. This result confirmed that the permeability increase in $\Delta$ tolpal mutants was not limited to PEN.

The increase in the outer membrane permeability of the $\Delta$ tol-pal mutants is growth phase-dependent

Recent studies showed that Tol-Pal complex is a part of cell division machinery, being involved in outer membrane invagination and modulation of peptidoglycan biosynthesis during the cell constriction. ${ }^{28,34}$ Establishing a method for the quantitative measurement of the outer membrane permeability of the $\Delta$ tol-pal mutant allowed us to examine the link between Tol-Pal function and cell division. With this aim, we examined the outer membrane permeability of $\Delta$ tol-pal mutants during the various growth phases using PEN as a substrate (Figure 2). We found that increases in outer membrane permeability were most pronounced in cells during the exponential growth phase, whereas it became almost equivalent to that of the parental strain during the stationary phase. There was no significant change in the permeability of $\Delta l p p$ or LA51 cells. These results suggest that only actively dividing $\Delta$ tol-pal mutant cells 
accompany outer membrane defects, supporting the proposed link between cell division and the outer membrane stabilizing effect by Tol-Pal function.

Increase of drug susceptibility in $\Delta t o l-p a l$ mutants, but not $\Delta l p p$ mutant, was almost comparable with that of $\triangle a c r A B$ mutant Although it is known that deleting tol-pal or $l p p$ leads to increased drug susceptibility, extent of the increase and the susceptibility pattern over various drugs has not been investigated in a comprehensive and quantitative manner using isogenic strains. The unexpectedly large

Table 1 Permeability coefficient of benzylpenicillin

\begin{tabular}{lc}
\hline Strain & Permeability coefficient $\left(\times 10^{-5} \mathrm{~cm} \mathrm{~s}^{-1}\right)^{\mathrm{a}}$ \\
\hline LA51 $\Delta$ acrAB & $0.07^{\mathrm{b}}$ \\
$\Delta$ tol $A$ acrAB & $0.23 \pm 0.06$ \\
$\Delta$ tolB $\Delta$ acrAB & $0.23 \pm 0.05$ \\
$\Delta$ tolQ $\Delta$ acrAB & $0.27 \pm 0.07$ \\
$\Delta$ tolR $\Delta$ acrAB & $0.29 \pm 0.05$ \\
$\Delta$ pal $\Delta$ acrAB & $0.30 \pm 0.07$ \\
$\Delta$ Ipp $\Delta$ acrAB & $0.08 \pm 0.03$ \\
\hline
\end{tabular}

${ }^{a}$ Mean \pm s.d. of triplicate measurements.

bData from ref. 15 .

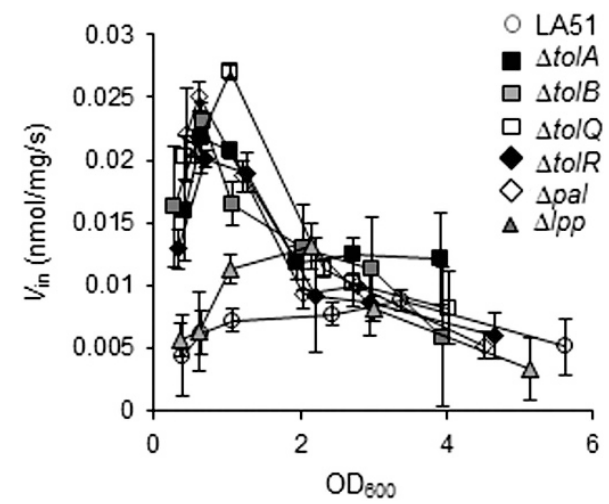

Figure 2 Rate of PEN influx into cells at various growth stages. $V_{\text {in }}$ was measured with $300 \mu \mathrm{m}$ of PEN. The data are expressed as the mean \pm s.d. of triplicate measurements from two independent experiments. increase in outer membrane permeability observed only in the $\Delta$ tol-pal mutants led us to investigate and determine the MICs for various drugs against each mutant in the presence or absence of $5 \mathrm{~mm}$ $\mathrm{MgCl}_{2}$ (Tables 2 and 3). We confirmed that there was no significant difference in the growth rates among the mutants in the absence of drugs (Supplementary Figure S1). To obtain the precise values of MIC, we used a gradient-plating method when the conventional serialdilution method failed to yield precise and reproducible MIC values. As expected, the $\Delta$ tol-pal mutants showed markedly reduced MICs for almost all of the antibiotics tested, regardless of their size or hydrophobicity. Ciprofloxacin was the exception, presumably reflecting the fact that fluoroquinolones can penetrate the outer membrane rapidly ${ }^{1}$ even in LA51 and thus the MIC was not affected significantly by the mutation that alters the outer membrane permeability. The MICs for the $\Delta$ tol-pal mutants were almost comparable with that of the $\triangle a c r A B$ mutant. By contrast, the MICs for $\Delta l p p$ were almost identical to those of the parental strain. These results confirmed that increased outer membrane permeability, as measured by PEN influx (Figure 1), correlated with drug susceptibility. These results also indicate that the phenotype of the $\Delta l p p$ mutant, at least in terms of outer membrane barrier function, was almost completely suppressed by $5 \mathrm{~mm} \mathrm{Mg}^{2+}$ (Table 2).

\section{Defining the outer membrane defect}

Measurement of the permeability of outer membrane diffusion channels in reconstituted liposomes. The pattern of drug susceptibility shown by the $\Delta$ tol-pal mutants was notable because increased susceptibility occurred regardless of the size and/or hydrophobicity of the drug. In general, defects in lipid bilayer do not significantly affect susceptibility to small, hydrophilic drugs, whereas defects in diffusion channels do not cause significant changes in susceptibility to large, hydrophobic drugs. $^{35,36}$ To clarify tol-pal specific outer membrane defect, we carried out (i) permeability measurement of liposomes reconstituted with outer membrane proteins and (ii) electron-microscopic observation of the cell surface structure. We reconstituted the outer membrane proteins from each mutant into liposomes and examined their permeability in a liposome-swelling assay ${ }^{23,37}$ using various sugars as substrates (Figure 3). We observed no significant difference between the LA51, $\Delta$ tol-pal and $\Delta l p p$ mutants. This suggests that the high level of outer membrane permeability observed in $\Delta$ tol-pal mutants was not due to proteinaceous factors, or at least not those that can be reconstituted into liposome. We examined the outer membrane

Table 2 MICs ( $\mu \mathrm{g} \mathrm{ml}^{-1}$ ) of various antibiotics determined in LB medium supplemented with $5 \mathrm{~mm} \mathrm{MgCl}_{2}$

\begin{tabular}{|c|c|c|c|c|c|c|c|c|c|}
\hline \multirow[b]{2}{*}{ Drug } & \multirow[b]{2}{*}{ Method } & \multicolumn{8}{|c|}{ Strain } \\
\hline & & LA51 & $\Delta$ tolA & $\Delta$ tolB & $\Delta$ tolQ & $\Delta$ tolR & $\Delta p a l$ & $\Delta / p p$ & $\triangle a c r A B$ \\
\hline Ampicillin & $\mathrm{G}$ & 44 & 2 & 2 & 1 & 2 & 2 & 44 & 3 \\
\hline Benzylpenicillin & G & 314 & 13 & 16 & 12 & 17 & 17 & 314 & 36 \\
\hline Carbenicillin & M & 32 & 8 & 8 & 8 & 2 & 4 & 32 & 4 \\
\hline Cloxacillin & $\mathrm{G}$ & $>500$ & 119 & 126 & 108 & 136 & 145 & $>500$ & $<1$ \\
\hline Cephalothin & M & 128 & 4 & 4 & 8 & 8 & 8 & 128 & 16 \\
\hline Tetracyclin & M & 8 & 4 & 4 & 4 & 4 & 4 & 8 & 2 \\
\hline Ciprofloxacin & M & 0.125 & 0.125 & 0.125 & 0.125 & 0.125 & 0.125 & 0.125 & 0.031 \\
\hline Vancomycin & M & 256 & 8 & 8 & 8 & 8 & 16 & 128 & 128 \\
\hline Novobiocin & $M$ & 64 & 32 & 32 & 32 & 16 & 32 & 128 & $<1$ \\
\hline Erythromycin & M & 128 & 16 & 32 & 16 & 16 & 32 & 64 & 8 \\
\hline Rifampicin & G & 10 & 1 & 1 & 1 & 1 & 1 & 5 & 3 \\
\hline
\end{tabular}

Abbreviations: G, determined using gradient-plating method; M, determined using microdilution method. 
Table 3 MICs ( $\mu \mathrm{g} \mathrm{ml}^{-1}$ ) of various antibiotics determined in LB medium without $\mathrm{MgCl}_{2}$

Strain

\begin{tabular}{|c|c|c|c|c|c|c|c|c|c|}
\hline Drug & Method & LA51 & $\Delta$ tolA & $\Delta t o l B$ & $\Delta t o / Q$ & $\Delta$ tolR & $\Delta p a l$ & $\Delta / p p$ & $\triangle a c r A B$ \\
\hline Ampicillin & $\mathrm{G}$ & 35 & 2 & 2 & 2 & 2 & 3 & 32 & 5 \\
\hline Benzylpenicillin & G & 333 & 24 & 27 & 27 & 30 & 35 & 320 & 49 \\
\hline Carbenicillin & M & 32 & 1 & 2 & 2 & 1 & 4 & 16 & 4 \\
\hline Cloxacillin & $G$ & $>500$ & 121 & 132 & 121 & 121 & 132 & $>500$ & $<1$ \\
\hline Cephalothin & M & 128 & 16 & 16 & 16 & 16 & 32 & 128 & 16 \\
\hline Tetracyclin & M & 0.5 & 0.5 & 0.5 & 0.5 & 0.5 & 0.5 & 1 & $<0.25$ \\
\hline Ciprofloxacin & M & 0.016 & 0.031 & 0.031 & 0.031 & 0.031 & 0.031 & 0.016 & $<0.008$ \\
\hline Vancomycin & M & 128 & 8 & 8 & $<4$ & $<4$ & $<4$ & 64 & 64 \\
\hline Novobiocin & M & 64 & 16 & 32 & 32 & 16 & 32 & 64 & $<1$ \\
\hline Erythromycin & M & 64 & 16 & 32 & 32 & 16 & 32 & 32 & 4 \\
\hline Rifampicin & $G$ & 10 & 1 & 1 & 1 & 1 & 1 & 6 & 6 \\
\hline
\end{tabular}

Abbreviations: G, determined using gradient-plating method; M, determined using microdilution method.
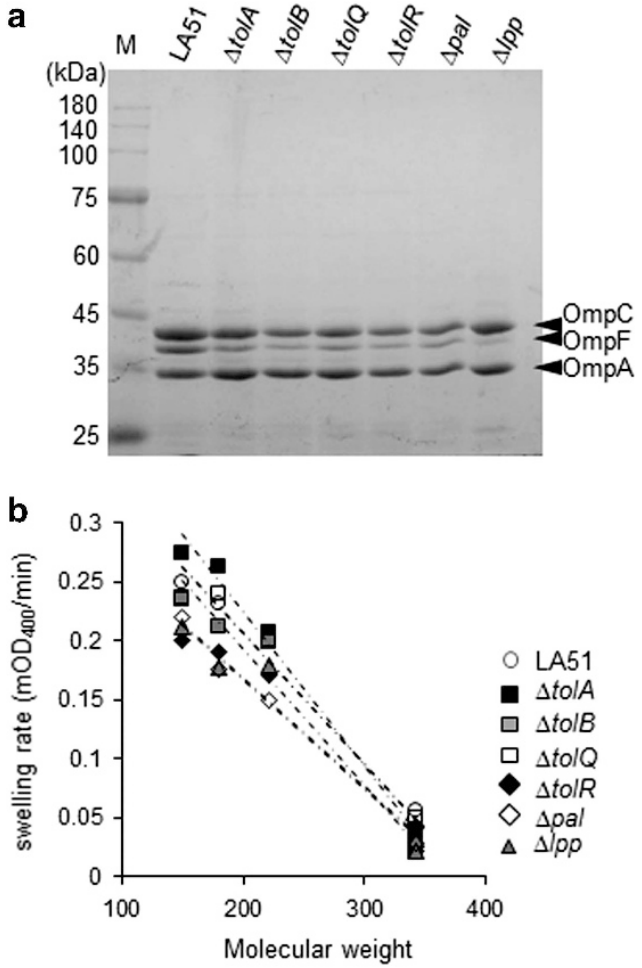

Figure 3 (a) Outer membrane proteins of LA51 and its derivative mutants. Outer membrane proteins were analyzed on 10\% SDS-PAGE gel containing $6 \mathrm{~m}$ urea. The gel was stained with Coomasie Brilliant Blue. M, molecular mass standards. (b) Liposome swelling assay. Outer membrane proteins $(2 \mu \mathrm{g})$ from LA51 and its derivative mutants were reconstituted into liposomes, and swelling rates were measured upon mixing with the substrates of different $\mathrm{MW}\left(M_{\mathrm{r}}\right)$. Substrates: arabinose $\left(M_{\mathrm{r}}\right.$ : 150$)$, glucose $\left(M_{\mathrm{r}}: 180\right)$, galactose $\left(M_{\mathrm{r}}: 180\right), N$-acetylglucosamine $\left(M_{\mathrm{r}}: 221\right)$ sucrose $\left(M_{\mathrm{r}}\right.$ : $342)$, lactose $\left(M_{r}: 342\right)$. The data are expressed as the mean of triplicate measurements.

proteins by SDS-PAGE (SDS polyacrylamide gel electrophoresis), but found no significant differences between the strains (Figure 3). As reported previously, ${ }^{38}$ the protein level of OmpF was slightly reduced in $\Delta$ tol-pal mutants, but this did not affect the permeability of proteoliposomes. In E. coli, the $\mathrm{OmpF}$ and $\mathrm{OmpC}$ porin channels are the main pathways through which hydrophilic solutes diffuse. ${ }^{1}$ The channel properties of these porins can be examined by measuring the influx rates of various substrates, that is, the rate of swelling of proteoliposome. We found that all proteoliposomes showed similar swelling rates and substrate size dependency. Thus, we concluded that deletion of the tol-pal or lpp genes had no effects on the function of the outer membrane diffusion channels.

Electron-microscopic observation of the cell surface structure. As there was no detectable defect in the outer membrane diffusion channels, another possible explanation for permeability increase should be physical instability of the membrane, which may result in transient fragility of the barrier. Although many studies have identified structural defects in the outer membrane of $\Delta$ tol-pal or $\Delta l p p$ mutants, ${ }^{8,13,24,39}$ none have addressed the specific defects or the preferential location of these defects for the $\Delta$ tol-pal mutants at the electron-microscopic level. We noted that the buffer used to measure the outer membrane permeability in intact cells (Figure 1) suppressed the leakage of $\beta$-lactamase for at least within $15 \mathrm{~min}$ at RT; nonetheless, there was a notable difference between the outer membrane permeability of the $\Delta t o l-p a l$ and $\Delta l p p$ mutant under this condition. We expected that electron-microscopic analysis under this condition would reveal $\Delta$ tol-pal specific defects in the outer membrane. To avoid the artificial damage during the sample preparation, we referred to the protocol of Fung et al., ${ }^{24}$ by which large blebs in the outer membrane in $\Delta l p p$ mutant grown in low- $\mathrm{Mg}^{2+}$ medium was observed. Accordingly, we performed all procedures at RT, and the cells were never subjected to centrifugal forces greater than $400 \mathrm{~g}$. With this sample, we observed the outer membrane defects specific for $\Delta$ tol-pal mutants (Figure 4). The outer membrane of the mutants lacking any component of Tol-Pal showed apparent rupture and/or large blebs at the cell division septum. Around $10 \sim 15 \%$ of the observed cells $(n \sim 30)$ showed this kind of defect. Blebs were also observed around the cell poles, although less frequently (around 6\%), presumably reflecting an inherited defect that might have originated at the site of constriction. There was no significant defect in the overall cell morphology of these mutants. On the other hand, no apparent ruptures, blebs or detachment from peptidoglycan were observed in the outer membrane of LA51 or the $\Delta l p p$ mutant. These results are consistent with the finding reported by Gerding et al., ${ }^{28}$ in which GFP protein was expressed in the periplasm of $\Delta$ tol-pal mutants, and an increase in periplasmic volume specifically at the site of constriction was observed. Taken together, the specific defect at the site of constriction, which presumably leads to structural instability of the 
a
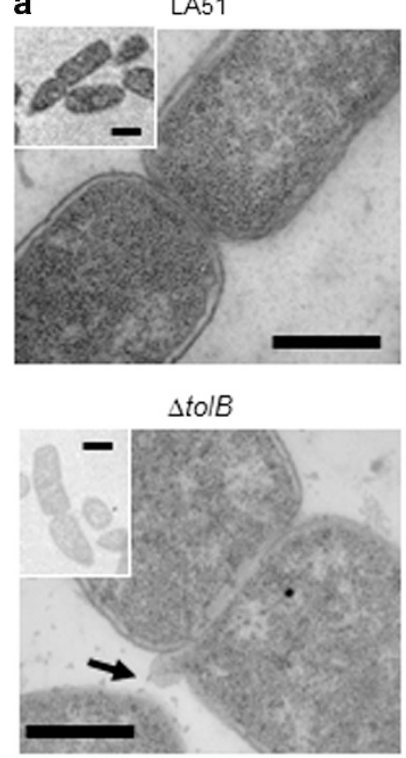

b

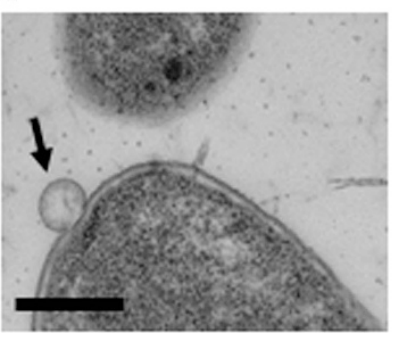

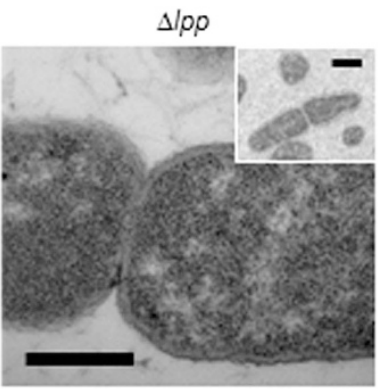
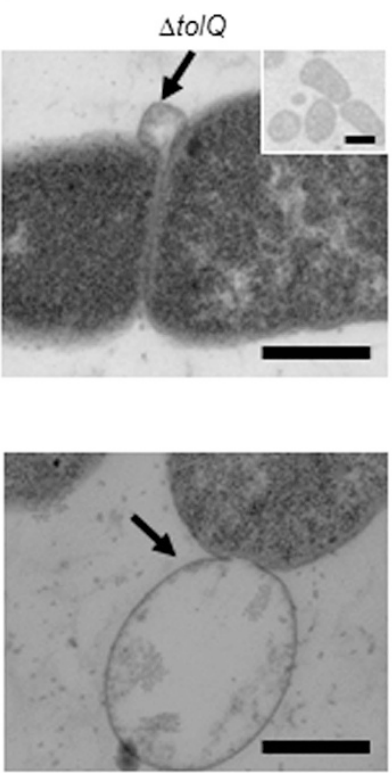
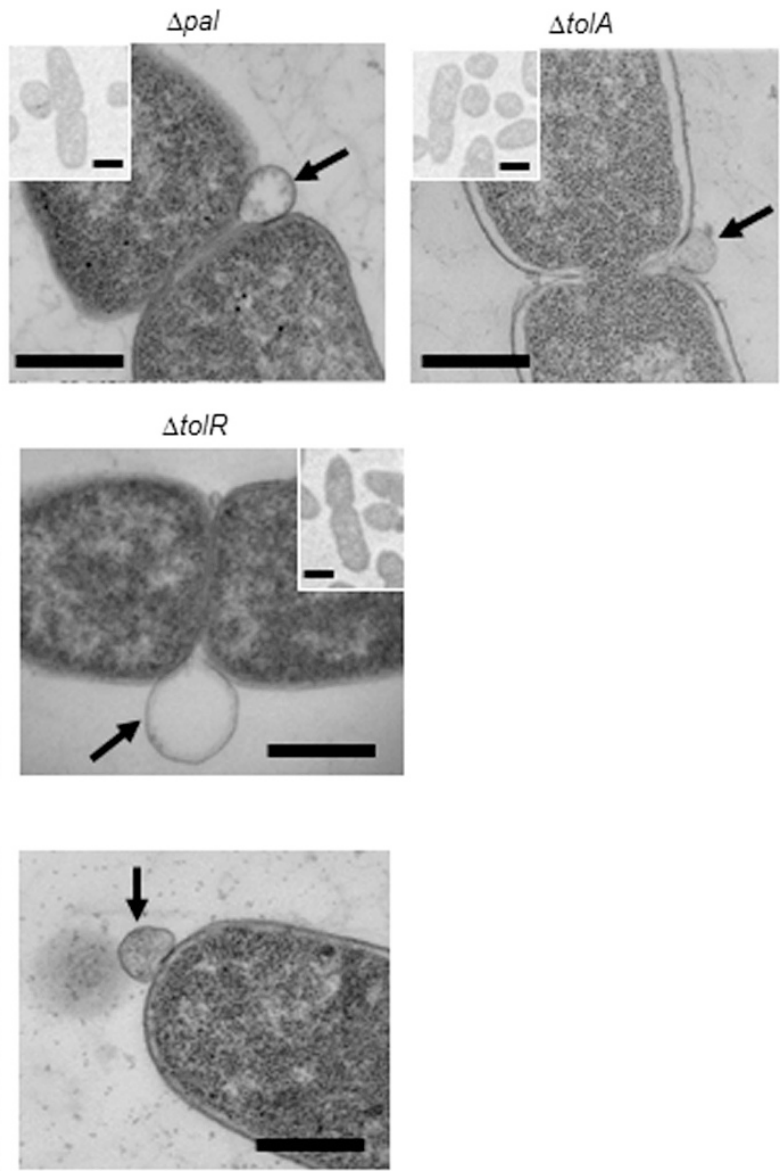

Figure 4 Cell surface structure of LA51 and its derivative mutants. Samples were stained with Ti blue and $0.4 \%$ lead citrate. (a) Cell constriction site. Structural defects in the outer membrane are indicated by arrows. Bars, $500 \mathrm{~nm}$. Overall morphology of the cells was shown in the inset. Bars in insets, $1 \mu \mathrm{m}$. (b) Cell poles. Three representative micrographs showing the cell poles of $\Delta$ tol-pal mutants are shown. Images show a $\Delta$ tolA mutant (left and center), and a $\Delta t o l B$ mutant (right).

outer membrane in $\Delta$ tol-pal mutants, appears most likely to explain the permeability increase in these strains.

\section{DISCUSSION}

The outer membrane of Gram-negative bacteria serves as a barrier that prevents the entry of toxic compounds into the cell. Thus, permeabilizing the outer membrane, if it is possible, is expected to be a promising strategy to combat multidrug-resistant Gram-negative bacteria. We determined that $\Delta$ tol-pal mutants, but not $\Delta l p p$ mutant, showed 3 - to 5 -fold increase in the outer membrane permeability compared with that of parental strain, and found that the drug susceptibility of $\Delta$ tol-pal mutants was almost comparable with that of $\triangle a c r A B$ mutant. We analyzed below the outer membrane barrier property and the drug susceptibility of $\Delta$ tol-pal mutants in comparison with that of $\triangle l p p$ and $\triangle a c r A B$ mutants.

\section{Comparison of tol-pal deletion and lpp deletion}

Cascales et al. showed that the outer membrane instability in $\Delta l p p$ mutant was rescued by overexpression of Pal protein, whereas overexpression of the Lpp protein did not suppress the $\Delta p a l$ phenotype. ${ }^{26}$ Gerding et al. ${ }^{28}$ revealed that Tol-Pal complex is a part of the cell division machinery and is required for proper invagination of the outer membrane during cell constriction, providing the evidence for Tol-Pal specific function. Our results demonstrated that the outer membrane barrier of $\Delta$ tol-pal mutants was indeed impaired only in the $\Delta$ tol-pal cells at exponential growth phase (Figure 2), supporting the proposed link between cell division and outer membrane stabilizing function by Tol-Pal.

A breach in the permeability barrier during cell constriction in $\Delta$ tol-pal mutants indicates that outer membrane integrity cannot be maintained just by passive tethering to peptidoglycan via Lpp, which distributes homogenously around the cell envelope. ${ }^{40}$ This may explain our observation that the permeability increase was evident only in $\Delta t o l-p a l$ mutants, but not in $\Delta l p p$ mutant, in the presence of $5 \mathrm{mM} \mathrm{MgCl}_{2}$. Loss of the tethering function of Lpp presumably led to only partial detachment of the outer membrane from the peptidoglycan; this might cause only limited instability that can be compensated for by the LPS-reinforcing properties of $\mathrm{Mg}^{2+}$. On the other hand, disorderly outer membrane invagination in $\Delta$ tol-pal mutants presumably causes more drastic outer membrane instability, as also evident by electron-microscopic observation (Figure 4), which could not be compensated for by $\mathrm{Mg}^{2+}$.

\section{Comparison of tol-pal deletion and $a c r A B$ deletion}

We showed that the drug susceptibility of $\Delta$ tol-pal mutants was almost comparable with that in the $\triangle a c r A B$ mutant (Table 2). This was not surprising considering that the major factors underlying the antibiotic resistance of Gram-negative bacteria are the outer 
membrane permeability barrier and efflux pump activity, and that these factors act synergistically. ${ }^{15,32,41}$ This synergistic relationship was indeed evident in Figure 1, as $V_{\text {in }}$ of PEN into $\Delta$ tol-pal mutants at low concentration $\left(C_{0}<200 \mu \mathrm{M}\right)$, a condition where the efflux activity by $A c r A B$ remains low because the periplasmic PEN concentration is low, ${ }^{15}$ was higher than that into $\triangle a c r A B$. This difference reflects the fact that $\Delta$ tol-pal mutants have defects in outer membrane barrier, on the other hand, the outer membrane of $\triangle a c r A B$ is intact.

The efficacy of small, hydrophilic drugs that rapidly permeate the outer membrane (for example, ampicillin), is less affected by efflux activity than that of bulky, hydrophobic drugs with low outer membrane permeability. ${ }^{42}$ The faster a drug permeates across the outer membrane, the more difficult it is for the efflux pump to counteract it. We revealed that the $P$-value for PEN in the $\Delta$ tol-pal mutants was 3- to 5-fold higher than that in the parental strain (Table 1). This allowed us to assess the impact of the permeability increase in a theoretical manner. PEN targets penicillin-binding proteins located in the periplasm. ${ }^{43}$ Thus, the level of resistance to PEN is dependent on the relationship between the PEN concentration in the periplasm $\left(C_{\mathrm{p}}\right)$ and that outside the cell $\left(C_{\mathrm{o}}\right)$; if $C_{\mathrm{p}}$ remains low against a high $C_{\mathrm{o}}$ level, it means that the cells are highly resistant to PEN. A theoretical $C_{\mathrm{p}}$ versus $C_{\mathrm{o}}$ curve is shown in Figure 5. This curve was created using kinetic parameters representing influx, efflux via AcrAB-TolC efflux pump, and hydrolysis via AmpC $\beta$-lactamase. ${ }^{20} \mathrm{We}$ assumed steady-state movement of PEN across the outer membrane, designated $V_{\text {in }}=V_{\mathrm{e}}+V_{\mathrm{h}}$, where $V_{\mathrm{in}}, V_{\mathrm{e}}$ and $V_{\mathrm{h}}$ are the rate of influx, efflux and hydrolysis, respectively (see 'Materials and Methods' section). ${ }^{15,33}$ A previous study showed that this steady-state assumption was applicable to explain the experimentally determined relationship between $C_{\mathrm{p}}$ and $C_{\mathrm{o}}$ relationship. ${ }^{15}$ As shown in the figure, assuming a 4 -fold increase in outer membrane permeability gave a $C_{\mathrm{p}}$ versus $C_{\mathrm{o}}$ curve similar to that for $\triangle a c r A B$ mutant, indicating that a 4 -fold increase was indeed sufficient to cause increased susceptibility to PEN at a level comparable with that conferred by the acr $A B$ deletion. Such a theoretical calculation is not possible for other classes of antibiotics because the kinetic parameters for influx and efflux are not available. However, considering that most antibiotics need to penetrate the periplasm to reach their targets, breaching the outer membrane barrier results in increased influx and an increase in $C_{\mathrm{p}}$,

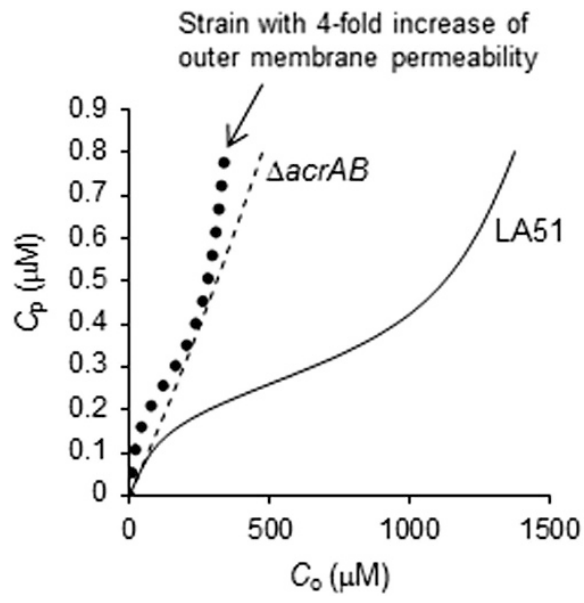

Figure 5 Theoretical relationship between the $C_{\mathrm{p}}$ and $C_{0}$ values for PEN in LA51 and its derivatives. Theoretical $C_{\mathrm{p}}$ versus $C_{0}$ curves for LA51 and the $\triangle a c r A B$ mutant are shown. The effect of increasing outer membrane permeability was assessed by assuming a 4-fold increase in the permeability coefficient. which ultimately results in higher drug concentration in the cytoplasm and increased susceptibility. Inhibiting multidrug efflux pumps is a promising way to combat multidrug-resistant Gram-negative bacteria. $^{32}$ Although direct inhibition of these pumps may be the most efficient strategy, our results show that inhibiting the function of the Tol-Pal complex can counteract efflux activity, thereby making this an alternative way to combat multidrug resistance.

\section{CONFLICT OF INTEREST}

The authors declare no conflict of interest.

\section{ACKNOWLEDGEMENTS}

We thank K Itoh for technical assistance with electron-microscopy.

1 Nikaido, H. Molecular basis of bacterial outer membrane permeability revisited. Microbiol. Mol. Biol. Rev. 67, 593-656 (2003).

2 Tokuda, H. Biogenesis of outer membranes in Gram-negative bacteria. Biosci. Biotechnol. Biochem. 73, 465-473 (2009).

3 Bos, M. P., Robert, V. \& Tommassen, J. Biogenesis of the gram-negative bacterial outer membrane. Annu. Rev. Microbiol. 61, 191-214 (2007).

4 Hirota, Y., Suzuki, H., Nishimura, Y. \& Yasuda, S. On the process of cellular division in Escherichia coli: a mutant of E. coli lacking a murein-lipoprotein. Proc. Natl Acad. Sci. USA 74, 1417-1420 (1977).

5 Braun, V. Covalent lipoprotein from the outer membrane of Escherichia coli. Biochim. Biophys. Acta. 415, 335-377 (1975).

6 Lazzaroni, J. C. \& Portalier, R. C. Isolation and preliminary characterization of periplasmic-leaky mutants of Escherichia coli K-12. FEMS Microbiol. Lett. 5, 411-416 (1979).

7 Suzuki, H. et al. Murein-lipoprotein of Escherichia coli: a protein involved in the stabilization of bacterial cell envelope. Mol. Gen. Genet. 167, 1-9 (1978).

8 Bernadac, A., Gavioli, M., Lazzaroni, J. C., Raina, S. \& Lloubes, R. Escherichia coli tol-pal mutants form outer membrane vesicles. J. Bacteriol. 180, 4872-4878 (1998).

9 Inouye, M., Shaw, J. \& Shen, C. The assembly of a structural lipoprotein in the envelope of Escherichia coli. J. Biol. Chem. 247, 8154-8159 (1972).

10 Godlewska, R., Wisniewska, K., Pietras, Z. \& Jagusztyn-Krynicka, E. K. Peptidoglycanassociated lipoprotein (Pal) of Gram-negative bacteria: function, structure, role in pathogenesis and potential application in immunoprophylaxis. FEMS Microbiol. Lett. 298, 1-11 (2009).

11 Lazzaroni, J. C., Dubuisson, J. F. \& Vianney, A. The Tol proteins of Escherichia coli and their involvement in the translocation of group A colicins. Biochimie 84 391-397 (2002).

12 Lloubes, R. et al. The Tol-Pal proteins of the Escherichia coli cell envelope: an energized system required for outer membrane integrity? Res. Microbiol. 152, 523-529 (2001).

13 Yem, D. W. \& Wu, H. C. Physiological characterization of an Escherichia coli mutant altered in the structure of murein lipoprotein. J. Bacteriol. 133, 1419-1426 (1978).

14 Jaurin, B., Grundstrom, T., Edlund, T. \& Normark, S. The E. coli beta-lactamase attenuator mediates growth rate-dependent regulation. Nature 290, 221-225 (1981).

15 Kojima, S. \& Nikaido, H. Permeation rates of penicillins indicate that Escherichia coli porins function principally as nonspecific channels. Proc. Natl Acad. Sci. USA 110, E2629-E2634 (2013).

$16 \mathrm{Baba}, \mathrm{T}$. et al. Construction of Escherichia coli K-12 in-frame, single-gene knockout mutants: the Keio collection. Mol. Syst. Biol. 2, 2006.0008 (2006).

$17 \mathrm{Kim}, \mathrm{H}$. S., Nagore, D. \& Nikaido, H. Multidrug efflux pump MdtBC of Escherichia coli is active only as a B2C heterotrimer. J. Bacteriol. 192, 1377-1386 (2010).

18 Datsenko, K. A. \& Wanner, B. L. One-step inactivation of chromosomal genes in Escherichia coli K-12 using PCR products. Proc. Natl Acad. Sci. USA 97, 6640-6645 (2000).

19 Millar, J. H. Experiments in Molecular Genetics (Cold Spring Harbor Laboratory Press, Cold Spring Harbor, 1972).

20 Kojima, S. \& Nikaido, H. High salt concentrations increase permeability through OmpC channels of Escherichia coli. J. Biol. Chem. 289, 26464-26473 (2014).

21 Novick, R. P. Micro-iodometric assay for penicillinase. Biochem. J. 83 236-240 (1962).

22 Smit, J., Kamio, Y. \& Nikaido, H. Outer membrane of Salmonella typhimurium: chemical analysis and freeze-fracture studies with lipopolysaccharide mutants. J. Bacteriol. 124, 942-958 (1975).

23 Nikaido, H., Nikaido, K. \& Harayama, S. Identification and characterization of porins in Pseudomonas aeruginosa. J. Biol. Chem. 266, 770-779 (1991).

24 Fung, J., MacAlister, T. J. \& Rothfield, L. I. Role of murein lipoprotein in morphogenesis of the bacterial division septum: phenotypic similarity of IkyD and Ipo mutants. J. Bacteriol. 133, 1467-1471 (1978). 
25 Nikaido, H., Bavoil, P. \& Hirota, Y. Outer membranes of gram-negative bacteria. XV. Transmembrane diffusion rates in lipoprotein-deficient mutants of Escherichia coli. J. Bacteriol. 132, 1045-1047 (1977).

26 Cascales, E., Bernadac, A., Gavioli, M., Lazzaroni, J. C. \& Lloubes, R. Pal lipoprotein of Escherichia coli plays a major role in outer membrane integrity. J. Bacteriol. 184, 754-759 (2002).

27 Nichols, R. J. et al. Phenotypic landscape of a bacterial cell. Cell 144, 143-156 (2011).

28 Gerding, M. A., Ogata, Y., Pecora, N. D., Niki, H. \& de Boer, P. A. The trans-envelope Tol-Pal complex is part of the cell division machinery and required for proper outer-membrane invagination during cell constriction in E. coli. Mol. Microbiol. 63, 1008-1025 (2007).

29 Lazzaroni, J. C. \& Portalier, R. C. Genetic and biochemical characterization of periplasmic-leaky mutants of Escherichia coli K-12. J. Bacteriol. 145, 1351-1358 (1981).

30 Nikaido, H. \& Normark, S. Sensitivity of Escherichia coli to various beta-lactams is determined by the interplay of outer membrane permeability and degradation by periplasmic beta-lactamases: a quantitative predictive treatment. Mol. Microbiol. 1, 29-36 (1987).

$31 \mathrm{Li}$, X. Z., Ma, D., Livermore, D. M. \& Nikaido, H. Role of efflux pump(s) in intrinsic resistance of Pseudomonas aeruginosa: active efflux as a contributing factor to beta-lactam resistance. Antimicrob. Agents Chemother. 38, 1742-1752 (1994).

32 Li, X. Z., Plesiat, P. \& Nikaido, H. The challenge of efflux-mediated antibiotic resistance in Gram-negative bacteria. Clin. Microbiol. Rev. 28, 337-418 (2015).

33 Nagano, K. \& Nikaido, H. Kinetic behavior of the major multidrug efflux pump AcrB of Escherichia coli. Proc. Natl Acad. Sci. USA 106, 5854-5858 (2009).
34 Gray, A. N. et al. Coordination of peptidoglycan synthesis and outer membrane constriction during Escherichia coli cell division. eLife 4, e07118 (2015)

35 Vaara, M. Agents that increase the permeability of the outer-membrane. Microbiol. Rev. 56, 395-411 (1992).

36 Vaara, M. Antibiotic-supersusceptible mutants of Escherichia coli and Salmonella typhimurium. Antimicrob. Agents Chemother. 37, 2255-2260 (1993).

37 Nikaido, H. \& Rosenberg, E. Y. Effect on solute size on diffusion rates through the transmembrane pores of the outer membrane of Escherichia coli. J. Gen. Physiol. 77, 121-135 (1981).

38 Lazzaroni, J. C., Fogninilefebvre, N. \& Portalier, R. C. Effects of Lkyb-mutations on the expression of Ompf, Ompc and Lamb Porin structural genes in Escherichia coli K-12. FEMS Microbiol. Lett. 33, 235-239 (1986).

39 Meury, J. \& Devilliers, G. Impairment of cell division in tolA mutants of Escherichia coli at low and high medium osmolarities. Biol. Cell 91, 67-75 (1999).

40 Hiemstra, H., Nanninga, N., Woldringh, C. L., Inouye, M. \& Witholt, B. Distribution of newly synthesized lipoprotein over the outer membrane and the peptidoglycan sacculus of an Escherichia coli lac-Ipp strain. J. Bacteriol. 169, 5434-5444 (1987).

$41 \mathrm{Lim}, \mathrm{S}$. P. \& Nikaido, H. Kinetic parameters of efflux of penicillins by the multidrug efflux transporter AcrAB-TolC of Escherichia coli. Antimicrob. Agents Chemother. 54, 1800-1806 (2010).

42 Mazzariol, A., Cornaglia, G. \& Nikaido, H. Contributions of the AmpC beta-lactamase and the AcrAB multidrug efflux system in intrinsic resistance of Escherichia coli K-12 to beta-lactams. Antimicrob. Agents Chemother. 44, 1387-1390 (2000).

43 Curtis, N. A., Orr, D., Ross, G. W. \& Boulton, M. G. Affinities of penicillins and cephalosporins for the penicillin-binding proteins of Escherichia coli K-12 and their antibacterial activity. Antimicrob. Agents Chemother. 16, 533-539 (1979).

Supplementary Information accompanies the paper on The Journal of Antibiotics website (http://www.nature.com/ja) 